This item was submitted to Loughborough's Research Repository by the author.

Items in Figshare are protected by copyright, with all rights reserved, unless otherwise indicated.

\title{
Considerations for single and double leg drop jumps: bilateral deficit, standardizing drop height, and equalizing training load
}

PLEASE CITE THE PUBLISHED VERSION

http://dx.doi.org/10.1123/jab.2014-0035

\section{PUBLISHER}

(c) Human Kinetics, Inc.

\section{VERSION}

AM (Accepted Manuscript)

\section{PUBLISHER STATEMENT}

This work is made available according to the conditions of the Creative Commons Attribution-NonCommercialNoDerivatives 4.0 International (CC BY-NC-ND 4.0) licence. Full details of this licence are available at: https://creativecommons.org/licenses/by-nc-nd/4.0/

\section{LICENCE}

CC BY-NC-ND 4.0

\section{REPOSITORY RECORD}

Pain, Matthew T.G.. 2019. "Considerations for Single and Double Leg Drop Jumps: Bilateral Deficit, Standardizing Drop Height, and Equalizing Training Load”. figshare. https://hdl.handle.net/2134/16688. 


\title{
Considerations for Single and Double Leg Drop Jumps: Bilateral Deficit, Standardising Drop Height and Equalising Training Load.
}

\author{
Matthew T.G. Pain
}

\begin{abstract}
School of Sport, Exercise and Health Sciences, Loughborough University, Loughborough,
\end{abstract} Leicestershire, UK.

\section{ABSTRACT}

Bilateral deficit is well documented; however, bilateral deficit is not present in all tasks and is more likely in dynamic activities than isometric activities. No definitive mechanism(s) for bilateral deficit is known but an oft cited mechanism is lower activation of fast twitch motor units. The aim of this study was to produce comparable and consistent one and two legged drop jumps to examine bilateral deficit in elite power athletes and elite endurance athletes. Seven power athletes and seven endurance athletes performed single and double leg drop jumps from a range of heights that equalised loading per leg in terms of: height dropped, energy absorbed, and momentum absorbed. Force and motion data were collected at $800 \mathrm{~Hz}$. Bilateral deficit for jump height, peak concentric force, and peak concentric power were calculated. Power athletes had a significantly greater $(p<0.05)$ bilateral deficit for jump height and peak power, possibly due to power athletes having more fast twitch motor units, however, endurance athletes generally had a bilateral surfeit which could confound this inference. Results indicate that equalizing loading by impulse per leg is the most appropriate and that a consistent drop height can be obtained with a short 10 minute coaching session.

Keywords: force plate, performance, sport, musculoskeletal

\section{INTRODUCTION}

Bilateral deficit has been the term applied to the inability to produce maximal voluntary force in both limbs simultaneously during bilateral actions ${ }^{1,2}$. There have been numerous studies providing evidence of bilateral deficit in both upper and lower limbs in a range of tasks. ${ }^{1}$ Tasks that have been investigated include: single joint isometrics ${ }^{3,4}$ single joint isokinetics, ${ }^{5}$ multi-joint isometrics, ${ }^{6,7}$ multi-joint isokinetic tasks, ${ }^{3}$ reflexively evoked contractions ${ }^{8}$ and dynamic skills such as jumping. ${ }^{9-11}$ Generally the difference between the sum of the unilateral and the bilateral measures is around $10 \%$ when bilateral deficit is present, but the presence of bilateral deficit can be task and subject specific. ${ }^{1}$

In the review by Jakobi and Chilibeck ${ }^{1}$ only around $70 \%$ of 42 studies investigating bilateral deficit provided support for it. These 42 studies covered 9 different tasks and included 31 isometric studies (upper and lower limbs) and 11 dynamic studies (upper and lower limbs and jumping). A bilateral deficit was reported in $21(70 \%)$ of the isometric studies and $9(82 \%)$ of the dynamic studies. Isometric knee extension was the only task where the majority of the studies reported no bilateral deficit, with five out of eight studies showing no bilateral deficit, whereas dynamic knee extension and combined knee-hip extension, even if performed isometrically, invariably demonstrated bilateral deficit. Recently Buckthorpe, Pain, \& Folland ${ }^{12}$ demonstrated high levels of bilateral deficit during explosive force production, even when absent in subsequent isometric maxima, thus it would appear that bilateral deficit is more commonly found, or its effects are more pronounced, during multi-joint and dynamic movements. 
Despite these numerous studies no definitive mechanism(s) responsible for bilateral deficit have been determined, although there are some agreements. The decrease in bilateral force is not due to increased antagonist activity. ${ }^{7,13}$ Bilateral deficit is influenced by training ${ }^{14,15}$ but in order to reduce bilateral deficit the training must be bilateral training, and commensurate with the testing protocol. ${ }^{16}$ The most often cited mechanism for bilateral deficit is a lesser activation of fast twitch motor units $^{2,13,17}$ but whether this originates due to inter-hemispheric inhibition, ${ }^{18}$ or at other supraspinal levels, ${ }^{16,19}$ or both, is not clear.

Since bilateral deficit is more commonly found during multi-joint and dynamic movements, drop jumps would appear a good candidate for testing for bilateral deficit. Drop jumps are a stretch-shorten cycle activity characterised by high muscular forces and velocities and drop jump ability is greatest amongst power athletes, who are considered to have a greater percentage of fast twitch motor units. $^{20,21}$ If bilateral deficit is due to lesser fast twitch motor recruitment and power athletes have a greater percentage of fast twitch motor units contributing to drop jumping ability than endurance athletes, then the power athletes should exhibit a greater degree of bilateral deficit in drop jumps. It is hypothesised that although elite power athletes will have greater jumping ability than elite endurance athletes, the power athletes will exhibit a greater degree of bilateral deficit in drop jumps. The aim of this study was to develop a method of producing comparable and consistent one and two legged drop jumps in order to examine bilateral deficit in elite power athletes and elite endurance athletes.

\section{METHODS}

Seven endurance athletes and seven power athletes (national and international level competitors, mean height $1.75 \pm 0.09 \mathrm{~m}$ and mean mass $72.5 \pm$ $10.2 \mathrm{~kg}$ ) performed a series of one legged and two legged drop jumps from three heights: $15 \mathrm{~cm}, 30 \mathrm{~cm}$ and $60 \mathrm{~cm}$, the $60 \mathrm{~cm}$ jumps were two legged only. All subjects gave informed consent and all protocols were approved by the University Ethical Advisory Committee. Subject numbers were determined from a power analysis based on literature values ${ }^{10,11}$ for bilateral deficits found in previous jumping studies (sample size calculated for an ANOVA gave a power of $>80 \%$ for 7 subjects, with a mean effect size 1.2, Cohen's d).

Drop jumps were performed with both the dominant and non-dominant legs for the single leg jumps. Drop jumps were performed without arm swing and with instruction to minimize the ground contact time in all jumps (bounce drop jumps) and the free leg usage in single leg jumps. Three drop jumps in each condition were performed. The order in which conditions were performed was randomly assigned and subjects were allowed as much recovery between trials as required, with a minimum of one minute. The highest drop jump for each condition in which the arm and free leg movement constraints were observed were selected for further analysis. The drop heights were chosen to allow the comparison of the single leg and double leg performances in terms of: same drop height, equal energy, and equal impulse per leg that had to be ameliorated during the eccentric phase $(15 \mathrm{~cm}$ single leg is equal energy per leg to $30 \mathrm{~cm}$ double leg, $30 \mathrm{~cm}$ single leg is equal energy per leg to $60 \mathrm{~cm}$ double leg, and $15 \mathrm{~cm}$ single leg is equal impulse per leg to $60 \mathrm{~cm}$ double leg).

All subjects were familiarized with the procedures in advance and were allowed to practice with instruction and feedback until the technique used was considered consistent and correct. This was necessary in order to standardize both 
dropping and jumping procedures so that bounce drop jumps from the required heights had landing velocities that reflected the theoretical landing velocities. This process was informed by an initial pilot study conducted in order to determine how to get consistent drop heights from the athletes. Eight subjects (mean height $1.70 \pm$ $0.12 \mathrm{~m}$ and mean mass $69.6 \pm 20.5 \mathrm{~kg}$, a mix of sedentary and athletic students) performed a total of 6 two legged and one legged drop landings off a box from 15 $\mathrm{cm}, 30 \mathrm{~cm}$ and $60 \mathrm{~cm}$ onto the Kistler force plate sampling at $1000 \mathrm{~Hz}$. All subjects gave informed consent and all protocols were approved by the University Ethical Advisory Committee. All landings finished with the subject standing upright with arms at their side. First this was done with no instruction then again after 10 minutes of coaching in how to step up and off so as not to let their centre of mass drop before leaving the takeoff block. Drop height of the centre of mass was calculated from the landing impulse and equations of uniformly accelerated motion.

For the main study force and 3-D movement data were recorded at $800 \mathrm{~Hz}$ using a Kistler 9281B12 force plate synchronized with two CODA mpx30 units. The drop jumps were also recorded at $50 \mathrm{~Hz}$ with a Sony digital camera to check that arm and free leg motions were as prescribed. All motion analysis calculations were performed in 2D for each side of the body. Active markers were placed at the following joint locations on both sides of the body: first metatarsal-phalangeal, lateral malleolus, knee, hip and shoulder. Motion data were reduced to a planar motion and low pass filtered at $8 \mathrm{~Hz}$ using a $4^{\text {th }}$ order zero lag Butterworth filter for subsequent analysis. Segment mass and centre of mass were calculated from segment lengths using Dempster. ${ }^{22}$ Whole body centre of mass was then calculated from segment data assuming a fixed torso, head, and shoulder-arm configuration. A random sample of two athletes was tested on a reaction board for the range of changes seen in the assumed fixed arm and upper body configuration on the video recordings and changes of $\leq 1.5 \mathrm{~cm}$ were found across both subjects. As the comparative analyses were within athlete the assumption of the fixed configuration per athlete was used throughout the analyses. Jump height after leaving the ground was calculated using the impulse from the force plate and equations of uniformly accelerated motion, along with the landing velocity of the centre of mass of the subject, obtained as above.

Bilateral deficit was calculated using dominant and non-dominant leg jump heights $\left(B L D_{J H}\right)$, peak concentric force $\left(B L D_{P F}\right)$, and peak concentric power $\left(B L D_{P P}\right)$ independently for each variable using equation 1.

$$
B L D_{x}=100 \times\left(1-\frac{B M}{D M+N D M}\right)
$$

eqn 1

Where: $\quad B L D_{x}=$ Bilateral Deficit

$\mathrm{DM}=$ Dominant leg Measure

$\mathrm{BM}=$ Bilateral leg Measure
$\mathrm{NDM}=$ Non-Dominant leg Measure

Normality was checked with a Lilliefors test. All datasets were normally distributed at the $\mathrm{P}<.050$ level, apart from $B L D_{P F}$ which was $P=.052$, and ANOVAS were used throughout. A series of ANOVAs were run each with a Tukey-Kramer pairwise comparison post hoc test if significance was found using Matlab (The MathWorks Inc., Natick, MA, USA) with significance set at 0.050 . For the pilot study a two by seven (independent variables, athlete type and drop type) ANOVA with the dependent variable of landing velocity was performed. In the main study two separate two by seven (independent variables, athlete type and drop type) ANOVA with the single dependent variable in each of foot contact time and jump height were 
performed. Also in the main study three separate two by five (independent variables, athlete type and drop height pairs) ANOVA with the single dependent variable in each of percentage bilateral deficit in jump height, peak concentric force and concentric power were performed. Finally a single one way ANOVA for the power athlete's data for drop height pairs with the dependent variable of jump height. Although corrections for multiple post hoc tests within an ANOVA were made there was no experiment wide correction made for running multiple different statistical tests. Drop height conditions are described in the results by combing drop height with the following abbreviations: $\mathrm{dl}=$ double leg, $s d=$ single leg dominant, $s n=$ single leg non-dominant

\section{RESULTS}

For the drop landings during the pilot study the pre-coached average error in drop height, the difference between the box height and the height the centre of mass actually dropped, was $12 \mathrm{~cm}$ across all drop heights. This corresponded to an average error of $40 \%$ as the relative error was generally greatest at the lowest drop heights due to subjects tending to drop their centre of mass before jumping by the same absolute amount regardless of starting height. Post-coaching the average error in drop height across all drop heights was $2.6 \mathrm{~cm}$, which corresponded to an average error of $9 \%$, and the relative error was consistent across all heights. Subsequently, in the main study there was no significant difference between power athletes and endurance athletes for landing velocities. There was a main effect of drop height but no interaction effect, thus the landing velocities were successfully controlled across both groups (Table 1).

As expected power athletes performed significantly better, jumping $35 \%$ to $50 \%$ higher than endurance athletes and having significantly shorter ground contact times (Table 2). There was also a significant difference between drop height conditions but no interaction effect for both jump height and foot contact time (Figure 1). For foot contact time $15 \mathrm{dl}$ and $30 \mathrm{dl}$ were significantly different from all other conditions, and for jump height the three bilateral jump heights were significantly different from the single leg jump heights.

Table 1. Theoretical and actual landing velocities for the Power Athletes (PA) and the Endurance Athletes (EA) from different pre-set drop heights after coaching.

\begin{tabular}{|c|c|c|c|}
\hline Drop type & $\begin{array}{c}\text { Theoretical } \\
\left(\mathrm{m}^{-1} \mathrm{~s}^{-1}\right)\end{array}$ & $\begin{array}{c}\text { Actual PA } \\
\left(\mathrm{m} \cdot \mathrm{s}^{-1}\right)\end{array}$ & $\begin{array}{c}\text { Actual EA } \\
\left(\mathrm{m} \cdot \mathrm{s}^{-1}\right)\end{array}$ \\
\hline $15 \mathrm{dl}$ & 1.72 & $1.99 \pm 0.12$ & $1.86 \pm 0.18$ \\
\hline $15 \mathrm{sd}$ & 1.72 & $1.70 \pm 0.08$ & $1.68 \pm 0.14$ \\
\hline $15 \mathrm{sn}$ & 1.72 & $1.70 \pm 0.07$ & $1.68 \pm 0.16$ \\
\hline $30 \mathrm{dl}$ & 2.43 & $2.51 \pm 0.11^{*}$ & $2.45 \pm 0.15^{*}$ \\
\hline $30 \mathrm{sd}$ & 2.43 & $2.28 \pm 0.11^{*}$ & $2.20 \pm 0.13^{*}$ \\
\hline $30 \mathrm{sn}$ & 2.43 & $2.31 \pm 0.21^{*}$ & $2.22 \pm 0.12^{*}$ \\
\hline $60 \mathrm{dl}$ & 3.43 & $3.38 \pm 0.05^{\#}$ & $3.28 \pm 0.08^{\#}$ \\
\hline
\end{tabular}

$\mathrm{dl}=$ double leg $\mathrm{sd}=$ single leg dominant $\mathrm{sn}=$ single leg non-dominant

* significantly different at the 0.05 level from all $15 \mathrm{~cm}$ drop heights and $60 \mathrm{~cm}$ drop heights.

\# significantly different at the 0.05 level from all $15 \mathrm{~cm}$ drop heights and $30 \mathrm{~cm}$ drop heights. Insert table 2 around here 


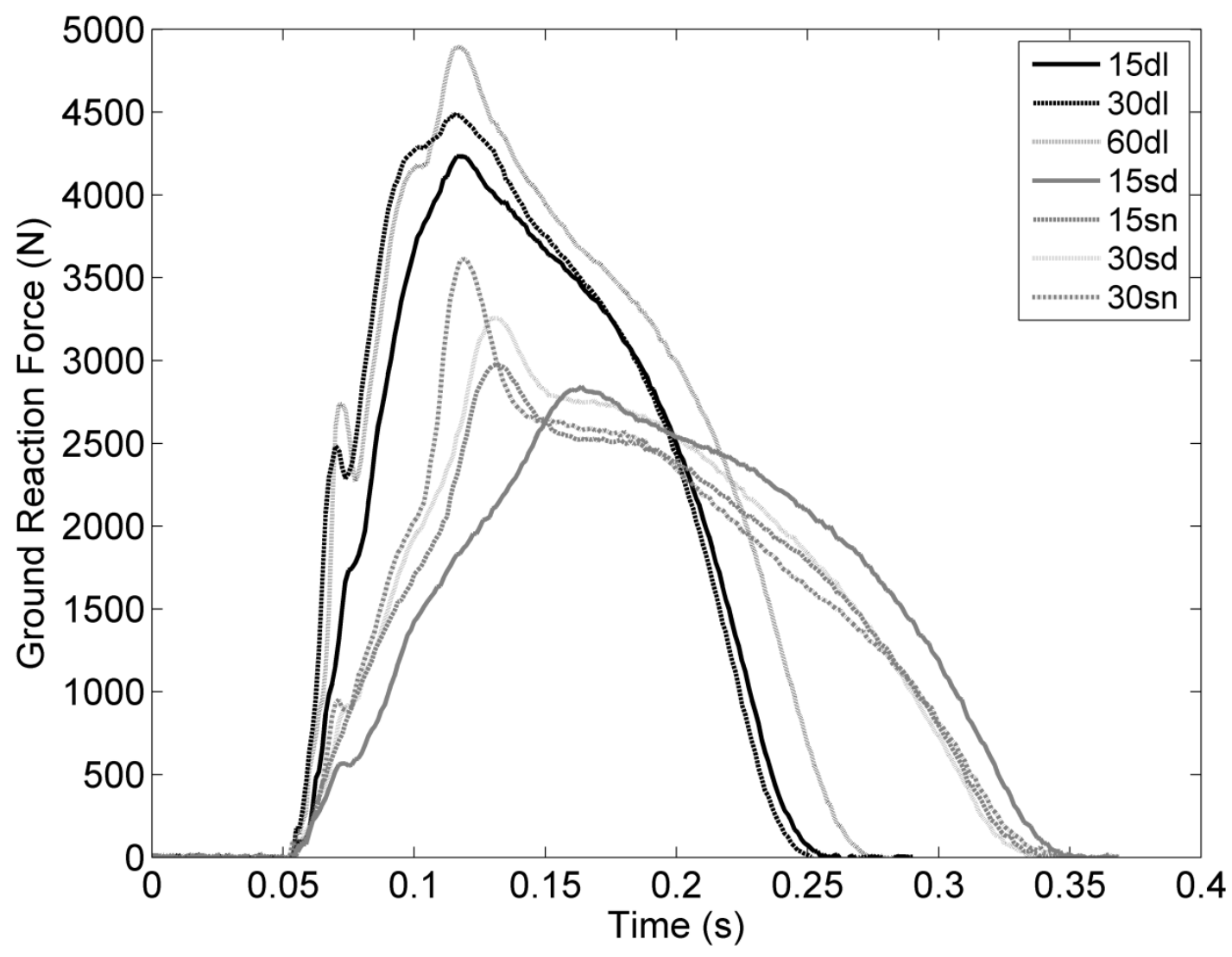

Figure 1. An exemplar set of ground reaction force time curves for one power athlete, showing all seven landing conditions. $15 \mathrm{dl}=15 \mathrm{~cm}$ drop bilateral, $30 \mathrm{dl}=30 \mathrm{~cm}$ drop bilateral, $60 \mathrm{dl}=60 \mathrm{~cm}$ drop bilateral, $15 \mathrm{sd}=15 \mathrm{~cm}$ drop dominant leg, $15 \mathrm{sn}=15 \mathrm{~cm}$ drop non-dominant leg, $30 \mathrm{sd}=30 \mathrm{~cm}$ drop dominant leg, $30 \mathrm{sn}=30 \mathrm{~cm}$ drop non-dominant leg.

Table 2. Drop jump performance results (mean and standard deviations); jump heights and contact times for the Power Athletes (PA) and the Endurance Athletes (EA), effect size is for between athlete group comparisons.

\begin{tabular}{|c|c|c|c|c|c|c|}
\hline $\begin{array}{l}\text { Drop } \\
\text { type }\end{array}$ & $\begin{array}{l}\text { Foot contact } \\
\text { time PA (s) }\end{array}$ & $\begin{array}{l}\text { Foot contact } \\
\text { time EA (s) }\end{array}$ & $\begin{array}{c}\text { Effect } \\
\text { size } \\
\text { Cohen's d }\end{array}$ & $\begin{array}{c}\text { Jump } \\
\text { height } \\
\text { PA (cm) }\end{array}$ & $\begin{array}{c}\text { Jump } \\
\text { height } \\
\text { EA }(\mathrm{cm})\end{array}$ & $\begin{array}{c}\text { Effect } \\
\text { size } \\
\text { Cohen's d }\end{array}$ \\
\hline 15dl & $\begin{array}{l}0.195 \pm \\
0.020^{\star \#}\end{array}$ & $\begin{array}{l}0.257 \pm \\
0.027^{\#}\end{array}$ & 2.61 & $\begin{array}{l}31.9 \pm \\
6.8^{\star \#}\end{array}$ & $22.8 \pm 7.8^{\#}$ & 1.24 \\
\hline 30dl & $\begin{array}{l}0.190 \pm \\
0.043^{\star \#}\end{array}$ & $\begin{array}{l}0.254 \pm \\
0.069^{\#}\end{array}$ & 1.11 & $\begin{array}{l}31.5 \pm \\
5.7^{\star \#}\end{array}$ & $24.2 \pm 7.6^{\#}$ & 1.09 \\
\hline $60 \mathrm{dl}$ & $\begin{array}{l}0.253 \pm \\
0.071^{*}\end{array}$ & $\begin{array}{c}0.273 \pm \\
0.054\end{array}$ & 0.32 & $\begin{array}{l}32.7 \pm \\
6.9^{\star \#}\end{array}$ & $24.6 \pm 9.8^{\#}$ & 0.96 \\
\hline $15 s d$ & $\begin{array}{l}0.258 \pm \\
0.032^{*}\end{array}$ & $\begin{array}{c}0.287 \pm \\
0.038\end{array}$ & 0.83 & $18.9 \pm 6.4^{*}$ & $12.5 \pm 4.4$ & 1.17 \\
\hline $15 s n$ & $\begin{array}{l}0.260 \pm \\
0.031^{*}\end{array}$ & $\begin{array}{l}0.291 \pm \\
0.029\end{array}$ & 1.03 & $18.1 \pm 5.7^{*}$ & $12.3 \pm 5.1$ & 1.07 \\
\hline 30sd & $0.291 \pm 0.042$ & $\begin{array}{l}0.300 \pm \\
0.046\end{array}$ & 0.20 & $17.1 \pm 6.2^{*}$ & $11.8 \pm 6.0$ & 0.87 \\
\hline 30sn & $\begin{array}{c}0.274 \pm \\
0.047^{\star}\end{array}$ & $\begin{array}{c}0.310 \pm \\
0.029\end{array}$ & 0.92 & $17.3 \pm 3.3^{*}$ & $11.2 \pm 6.3$ & 1.21 \\
\hline
\end{tabular}

$\mathrm{dl}=$ double leg $\mathrm{sd}=$ single leg dominant $\mathrm{sn}=$ single leg non-dominant

Between athlete groups: * significantly different from EA, at 0.05 level, from equivalent drop height.

Between drop heights: ${ }^{\#}$ significantly different, at 0.05 level, from all other drop height conditions. 
As hypothesized, power athletes exhibited a much larger bilateral deficit in all combinations of drop height when using jump height as a measure (Table 3), with a significant main effect of athlete group. There was also a significant effect of drop height on BLD $\mathrm{JH}_{\mathrm{H}}$, with $30 \mathrm{dl}-30 \mathrm{sl}$ and $60 \mathrm{dl}-30 \mathrm{dl}$ lower than all other drop conditions, as well as a significant interaction effect. However, what was less expected was the observation that endurance athletes demonstrated a bilateral surfeit, i.e. their two legged jumps were more than twice the height of their one legged jumps, in all but one condition. It should be noted that levels of $B L D_{J H}$ were very varied across endurance athletes, especially when calculations from $30 \mathrm{~cm}$ single leg jumps were involved. Due to the large surfeit values for endurance athletes a one way ANOVA for power athletes only showed that 30dl-30sl was significantly lower than all other drop heights and $60 \mathrm{dl}-15 \mathrm{sl}$ was higher than all others. Both groups displayed large $B L D_{P F}$, with a significant difference between groups, but not by drop height, and there was no interaction effect. However, the results for BLDPP show the same deficit levels between groups as for jump height (Table 4) and had a significant difference between groups, but not by drop height and there was no interaction effect.

Table 3. Bilateral deficit for jump heights for the Power Athletes (PA) and the Endurance Athletes (EA), negative values indicate a bilateral surfeit (mean and standard deviations), effect size is for between athlete group comparisons.

\begin{tabular}{|c|c|c|c|}
\hline $\begin{array}{c}\text { Drop height } \\
\text { pairs }\end{array}$ & $\begin{array}{c}\text { \% deficit PA } \\
\text { jump height }\end{array}$ & $\begin{array}{c}\text { \% deficit EA } \\
\text { jump height }\end{array}$ & $\begin{array}{c}\text { Effect size } \\
\text { Cohen's d }\end{array}$ \\
\hline 30dl-30sl & $1.60 \pm 15.1^{* \#}$ & $-27.6 \pm 37.7^{\#}$ & 1.02 \\
\hline $15 \mathrm{dl}-15 \mathrm{sl}$ & $6.90 \pm 17.9^{*}$ & $1.50 \pm 8.90$ & 0.38 \\
\hline $60 \mathrm{dl}-30 \mathrm{sl}$ & $7.80 \pm 13.4^{\star \#}$ & $-33.2 \pm 35.5^{\#}$ & 1.53 \\
\hline $30 \mathrm{dl}-15 \mathrm{sl}$ & $12.6 \pm 10.5^{*}$ & $-4.30 \pm 8.80$ & 1.74 \\
\hline $60 \mathrm{dl}-15 \mathrm{sl}$ & $17.7 \pm 12.2^{*}$ & $-9.60 \pm 13.3$ & 2.14 \\
\hline
\end{tabular}

$\mathrm{dl}=$ double leg $\mathrm{s}=$ single leg

Between athlete groups: * significantly different from EA, at 0.05 level, from equivalent drop height. Between drop heights: " significantly different, at 0.05 level, from all other drop height conditions.

Table 4. Bilateral deficit for peak concentric force and peak concentric power for the Power Athletes (PA) and the Endurance Athletes (EA) (mean and standard deviationsfor), effect size is for between athlete group comparisons.

\begin{tabular}{|c|c|c|c|c|c|c|}
\hline $\begin{array}{c}\text { Drop height } \\
\text { pairs }\end{array}$ & $\begin{array}{c}\text { \% deficit } \\
\text { PA Force }\end{array}$ & $\begin{array}{c}\text { \% deficit } \\
\text { EA Force }\end{array}$ & $\begin{array}{c}\text { Effect size } \\
\text { Cohen's d }\end{array}$ & $\begin{array}{c}\text { \% deficit } \\
\text { PA Power }\end{array}$ & $\begin{array}{c}\text { \% deficit } \\
\text { EA Power }\end{array}$ & $\begin{array}{c}\text { Effect size } \\
\text { Cohen's d }\end{array}$ \\
\hline 30dl-30sl & $16.8 \pm 10.1^{*}$ & $28.2 \pm 11.6$ & 1.05 & $1.1 \pm 17.4^{*}$ & $-13.8 \pm 16.3$ & 0.88 \\
\hline 15dl-15sl & $25.1 \pm 2.4^{*}$ & $32.8 \pm 8.6$ & 1.22 & $-4.8 \pm 20.5$ & $1.7 \pm 8.1$ & -0.42 \\
\hline $60 \mathrm{dl}-30 \mathrm{sl}$ & $28.3 \pm 15.8^{*}$ & $34.2 \pm 13.6$ & 0.40 & $5.8 \pm 12.8^{*}$ & $-3.8 \pm 17.6$ & 0.62 \\
\hline 30dl-15sl & $21.8 \pm 8.8^{*}$ & $29.7 \pm 13.3$ & 0.70 & $4.7 \pm 16.3^{*}$ & $-5.3 \pm 12.7$ & 0.68 \\
\hline $60 \mathrm{dl}-15 \mathrm{sl}$ & $32.9 \pm 15.8$ & $35.5 \pm 15.9$ & 0.16 & $11.2 \pm 11.4^{*}$ & $2.4 \pm 11.4$ & 0.77 \\
\hline
\end{tabular}

$\mathrm{dl}=$ double leg $\mathrm{sl}=$ single leg

Between athlete groups: * significantly different from EA, at 0.05 level, from equivalent drop height.

\section{DISCUSSION}

The main results of this study support the hypothesis that although elite power athletes have greater jumping ability than elite endurance athletes, the power athletes exhibit a greater degree of bilateral deficit in drop jumps. For both power athletes and endurance athletes, BLD $\mathrm{JH}_{\mathrm{H}}$ and BLD $\mathrm{PP}_{\mathrm{PP}}$ had similar overall patterns but the average $B L D_{\mathrm{PP}}$ was only $\sim 40 \%$ of $B L D_{\mathrm{JH}}$. The results for $B L D_{\mathrm{PF}}$ were completely different from those of jump height and peak concentric power. BLD $\mathrm{PF}_{\mathrm{F}}$ was similar 
for both groups of athletes with both showing a very large bilateral deficit. The peak concentric force always occurred at the start of the concentric phase as the force time curve transitioned from the high force eccentric braking phase to the concentric propulsive phase, and so would not necessarily be expected to be indicative of jumping performance. For power athletes the average BLD $\mathrm{JH}_{\mathrm{H}}$ across all drop

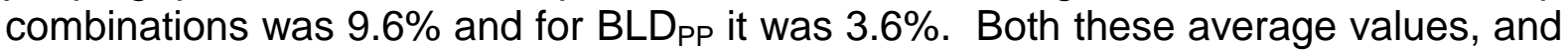
indeed values from all drop height combinations that showed a bilateral deficit, fall within the range reported in the literature for bilateral deficit associated with dynamic actions, with the $9.6 \%$ being close to the average literature values ${ }^{1}$.

What is surprising is the large bilateral surfeit in $B L D_{J H}$ found for the endurance athletes, on average a score of $-14.6 \%$ across all drop combinations, and even with the two $30 \mathrm{~cm}$ single leg drops removed there is still an average surfeit of $4.1 \%$. Reports of bilateral surfeits, or facilitations, are uncommon. The few studies reporting them have used elite athlete groups with a specific expertise in the bilateral task and the bilateral surfeit was at lower levels than seen here. ${ }^{6,15,23}$ Given that the endurance athletes were not specifically skilled at either single or double legged drop jumps, and their normal actions are unilateral, it is likely that this large surfeit has arisen due to problems with the drop jumping protocol, such as controlled single leg jumping and the drop heights chosen, in this group.

Drop jumps can produce greater jump heights than conventional jumps, however, after a certain threshold landing velocity there is a decrease in performance, so some care must be taken in their use in testing and in training. When comparing one legged and two legged drop jumps in an experiment investigating bilateral deficit it is necessary to avoid overloading a limb on landing in one condition and not in another. If a limb is overloaded in the eccentric phase to the extent that it is unable to produce a valid jump attempt in the concentric phase a fair comparison of determine bilateral deficit cannot be made. The results indicate that the endurance athletes were unable to perform successful single leg drop jumps from a height of $30 \mathrm{~cm}$. It is likely that even the 15s condition was too high for many of the endurance athletes and little or no drop jump benefit was gained, at least relative to any bilateral drop jump performance gains they may have had. However, given the results here it would be hard to see a combination of functional drop heights that would have led to a high $B L_{J H}$ value commensurate with those observed for the power athletes group. Recently it has been suggested that bilateral deficit may stem from the inability to fully utilise stabiliser muscles. ${ }^{12,24}$ Stimulated quadriceps activation, via the femoral nerve, demonstrated bilateral deficit in rate of force development measures ${ }^{12}$ and indicated that at least some of the bilateral deficit effects are not due to neural drive or agonist muscle properties and hence other mechanical factors play a part. In this study the coordinated movements required to produce the jump have a wider scope for stabilisation effects, and transfer of power between segments to positively or negatively affect single and double leg jumps to different extents.

As bilateral deficit is generally ascribed to the inability to produce maximal force some questions have been raised as to the validity of using the dynamic multijoint task of jumping performance as an indicator of bilateral deficit. Although jump height does not correlate well with maximal isometric force, and is a skilled activity, it does correlate with maximal power ${ }^{25}$ Challis $^{10}$ used a computer model of drop jumping to show that without allowing for bilateral deficit single leg jump height was $51.1 \%$ of double leg jump height. Allowing for a bilateral deficit factor it was $58.8 \%$, which compared favourably with his subjects values of $58.1 \%$ (these are equivalent 
to 13.9 and $14.9 \%$ deficit values using the calculation method in this paper). Bobbert et al. ${ }^{11}$ also used a modelling approach to state that $~ 75 \%$ of the bilateral deficit in work per leg throughout the entire concentric phase in squat jumping in the model was due to the higher shortening velocities and the other $25 \%$ was due to lower activation levels and hence there was no separate bilateral deficit phenomenon. However, although the results of Bobbert et al. ${ }^{11}$ did indeed show that the model performances could be explained without recourse to a separate bilateral deficit phenomenon the model did not compare well with the subjects' results it was based on. The model matched the mean of the subjects two legged jump height measured from standing height to within a millimetre $(28.3 \pm 6 \mathrm{~cm}$ versus $28.4 \mathrm{~cm})$ but the single leg jump for the model was $3.1 \mathrm{~cm}$ lower than the mean of the subjects $(19.3 \pm$ $5.1 \mathrm{~cm}$ versus $16.2 \mathrm{~cm}$ ), i.e. the subjects demonstrated a much greater BLD ${ }_{\mathrm{JH}}$ $(26.4 \%)$ than the model $(12.7 \%)$. If the measure of jump height is taken as the height the centre of mass is raised during the flight phase only, as in Challis ${ }^{10}$ and in this study then the subjects in Bobbert et al. ${ }^{11}$ have a BLDJH of around $5 \%$ and the model has a BLD $\mathrm{JH}_{\mathrm{H}}$ of $-33 \%$, a large surfeit. As such it would appear that the single leg jumping model in Bobbert et al. ${ }^{11}$ was not necessarily a good representation of single leg jumping and the appropriateness of using jumping as a means of investigating bilateral deficit remains equivocal.

It was intended that by using a range of drop heights this study could indicate whether drop height should be equalised in terms of impulse or energy per leg in order to make valid comparisons of bilateral deficit without the confounding effect of unequal drop jump facilitation. This could also aid with understanding how to optimally transfer between single and double leg drop jumps during training. Knowing the equivalent training load when moving between single and double leg jumps would be beneficial, especially when looking at progressive overload. The pilot study did determine that the accuracy of the drop jump drop height could be significantly improved with minimal coaching and guidance by reducing pre-emptive dropping of the centre of mass before stepping off the take-off block. Subjects tended to drop their centre of mass lower before take-off for single leg drop jumps compared to double leg drop jumps. It was noted that nearly all subjects from all groups had this characteristic when they first performed single leg drop jumps. Coupling this with a likely bilateral deficit seen in the power athletes, who will utilise this type of training, means they are reducing the 'landing load difficulty' considerably from that expected from halving the drop height. For both subject groups the foot contact time for $60 \mathrm{dl}$ was most similar to the $15 \mathrm{sd}$ and $15 \mathrm{sn}$ times and significantly different from the $15 \mathrm{dl}$ and $30 \mathrm{dl}$, indicating a distinct change in performance with regard to this parameter. Further for the power athletes, the BLD ${ }_{J H}$ for $30 \mathrm{dl}-30 \mathrm{sl}$ being significantly lower than the other conditions could be due to $30 \mathrm{~cm}$ providing good facilitation for double leg jumps but being poorer at facilitating one legged jumps. It is clear from the endurance athletes data that $30 \mathrm{~cm}$ single leg drop jumps were markedly inferior to all other heights including the $60 \mathrm{dl}$ for this group. Also in this study the $30 \mathrm{~cm}$ single leg drop jumps were deemed the most strenuous by the subjects, much more so than the $60 \mathrm{~cm}$ double leg drop jumps. In this study, with the drop heights well controlled, it seems that equalising by impulse gives the more equivalent conditions for comparing one and two legged performance, and may be what athletes during training are attempting when they drop their centre of mass before one legged drops.

As with all studies there are some limitations, such as the small sample size as it focused on getting high level subjects in each group to maximise between group 
differences. Although a power analysis, and previous experimental results, indicated that seven subjects would be sufficient this was based on calculating power for an ANOVA when only including the main effects and so may have had insufficient power to determine interaction effects. Also although within an ANOVA correction for multiple post hoc tests was made no across experiment correction was made for multiple tests. Not all statistical tests performed would fall under the same family but a number would, and special care should be taken with interpreting any single result from the first and second tests where power athletes were also tested separately from the pooled subjects. However, overall no single statistically significant result was used to come to a conclusion, but rather the general implications from a number of significant results were used to inform the conclusions drawn in this study. A further limitation of low subject numbers is the difficulty in generalising results to wider populations, especially in this case where the two groups showed such distinct performance differences. As well as statistical analysis limitations there are other experimental ones, such as not being able to fully control the free leg in the single leg jumps. Not only can changes in centre of mass of the limb aid in jump height when it is moved, the free limb motion can also aid jump height by contributing extra energy to the whole system and this was not quantified. Secondly optimal drop heights were not used as previously discussed."

Although the main hypothesis was supported with greater bilateral deficit present in the power athletes than endurance athletes this could be due to the bilateral surfeit in endurance athletes having arisen due to the drop jumping tasks being too difficult for them. This is despite different combinations of equalising the loading per leg during the drop landing phase. The study also highlighted the need to carefully control drop jump dropping protocols in order to maintain the actual subject drop height to a consistent value close to the desired height. Not controlling this could potentially confound comparisons between single and double leg drop jumps in testing and training. Therefore indirect support from this study would indicate that equalising by impulse per leg would be most appropriate as a first approximation then some small changes could also be made to account for bilateral deficit. 


\section{References}

1. Jakobi JM, Chilibeck PD. Bilateral and unilateral contractions: possible differences in maximal voluntary force. Can J Appl Physiol. 2001;26;12-33.

2. Vandervoort AA, Sale DG, Moroz J. Comparison of motor unit activation during unilateral and bilateral leg extension. J Appl Physiol. 1984;56:46-51.

3. Koh, T. J., Grabiner, M. D., \& Clough, C.A. (1993). Bilateral deficit is larger for step than for ramp isometric contractions. J Appl Physiol. 1993;74:1200-1205.

4. Schantz PG, Moritani T, Karlson E, et al. Maximal voluntary force of bilateral and unilateral leg extension. Acta Physiol Scand. 1989;136:185-192.

5. Cresswell AG, Ovendal AH. Muscle activation and torque development during maximal unilateral and bilateral isokinetic knee extensions. J Sport Med Phys Fit. 2002;42:19-25.

6. Secher $\mathrm{NH}$. Isometric rowing strength of experienced and inexperienced oarsmen. Med Sci Sport. 1975;7:280-283.

7. Behm DG, Power KE, Drinkwater EJ. Muscle activation is enhanced with multiand uni-articular bilateral versus unilateral contractions. Can J Appl Physiol. 2003;28:38-52.

8. Khodiguian N, Cornwell A, Lares $E$, et al. Expression of the bilateral deficit during reflexively evoked contractions. J Appl Physiol. 2003;94:171-178.

9. van Soest AJ, Roebroeck ME, Bobbert M.F, et al. A comparison of one-legged and two-legged countermovement jumps. Med Sci Sport Exer. 1985;17:635639.

10. Challis $\mathrm{JH}$. An investigation of the influence of bi-lateral deficit on human jumping. Hum Movement Sci. 1998;17:307-325.

11. Bobbert MF, de Graaf WW, Jonk JN,Casius LJ. Explanation of the bilateral deficit in human vertical squat jumping. J Appl Physiol.2006;100:493-499.

12. Buckthorpe MW, Pain MTG, Folland JP. Bilateral deficit in explosive force production is not caused by changes in agonist neural drive. PLOS ONE. 2013;8(3): e57549. doi:10.1371/journal.pone.0057549.

13. Kawakami Y, Sale DG, MacDougall JD, Moroz JS. Bilateral deficit in plantar flexion: relation to knee joint position, muscle activation, and reflex excitability. Eur J Appl Physiol O. 1998;77:212-216.

14. Secher NH, Rube N, Elers J. Strength of two- and one-leg extension in man. Acta Physiol Scand. 1988;134:333-339.

15. Howard JD, Enoka RM. Maximum bilateral contractions are modified by neurally mediated interlimb effects. J Appl Physiol.1991;70(1):306-316.

16. Taniguchi $Y$. Relationship between the modifications of bilateral deficit in upper and lower limbs by resistance training in humans. Eur $J$ Appl Physiol $O$. 1998;78:226-230.

17. Oda S, Moritani T. Maximal isometric force and neural activity during bilateral and unilateral elbow flexion in humans. Eur J Appl Physiol O. 1994;69(3):240243.

18. Ohtsuki T. Decrease in human voluntary isometric arm strength induced by simultaneous bilateral exertion. Behav Brain Res. 1983;7:165-178.

19. Post $M$, van Duinen $H$, Steens $A$, et al. Reduced cortical activity during maximal bilateral contractions of the index finger. Neuroimage. 2007;35:16-27.

20. Costill DL (1976). The relationship between selected physiological variables and distance running performance. J Sport Med Phys Fit.1976;7:610-616.

21. Viitasalo JT, Salo A, Lahtinen J. Neuromuscular functioning of athletes and nonathletes in the drop jump. Eur J Appl Physiol O. 1998;78:5432-5440. 
22. Dempster WT. Space Requirements of the seated operator. Wright-Patterson Air Force Base, Ohio. 1955 (WADCTR-55-159.)

23. Ebben WP, Flanagan $E$, Jensen $R L$. Bilateral facilitation and laterality during the countermovement jump. PerceptMotor Skill. 2009;108:251-2588.

24. Magnus CR, Farthing JP. Greater bilateral deficit in leg press than in handgrip exercise might be linked to differences in postural stability requirements. Appl Physiol Nutr Metab. 2008;33:1132-1139.

25. Zatsiorsky VM. Science and practice of strength training. Champaign, IL: Kinetic Books; 1995. 